\title{
Electromagnetoelastic actuator for large telescopes
}

\begin{abstract}
The structural diagram and the transfer functions, the characteristics of the electromagnetoelastic actuator for the large telescopes and the cosmic telescopes are obtained. The generalized structural diagram, the matrix transfer functions of the electromagnetoelastic actuator are described the characteristics of the actuator with regard to its physical parameters and external load.
\end{abstract}

Keywords: electromagnetoelastic actuator, nano-and microdisplacement, electromagnetoelasticity, structural diagram, piezoactuator, transfer function
Volume 2 Issue 5 - 2018

\author{
Afonin SM \\ National Research University of Electronic Technology (MIET), \\ Russia
}

Correspondence: Afonin SM, National Research University of Electronic Technology (MIET), Moscow, Russia, Email learner0।@mail.ru

Received: August 17,2018 | Published:September 18,2018

\section{Introduction}

The electromagnetoelastic actuator for the nano- and microdisplacement on the piezoelectric, piezomagnetic, electrostriction, magnetostriction effects is used in the electromechanics systems for the large telescopes and for the cosmic telescopes. ${ }^{1-8}$ The mathematical model, the structural diagram and transfer functions of the electromagnetoelastic actuator are calculated for designing the control system for the large telescopes and the adaptive optics. ${ }^{9-18}$ The structural diagram and transfer functions the electromagnetoelastic actuator based on the electromagnetoelasticity make it possible to describe the dynamic and static properties of the electromagnetoelastic actuator for the large telescopes, the cosmic telescopes and the adaptive optics with regard to its physical parameters and external load. ${ }^{19-22}$

\section{Structural diagram}

The structural diagram of the electromagnetoelastic actuator for the large telescopes, the cosmic telescopes and the adaptive optics is changed from Cady and Mason electrical equivalent circuits.

The method of mathematical physics with Laplace transform is applied for the solution the wave equation and for the determination the structural diagram of the electromagnetoelastic actuator for the nano- and microdisplacement. ${ }^{1-18}$

The generalized equation of the electromagnetoelasticity ${ }^{8,11,14}$ has the following form

$$
S_{i}=v_{m i} \Psi_{m}(t)+s_{i j}^{\Psi} T_{j}(x, t)(1)
$$

where $S_{i}=\partial \xi(x, t) / \partial x$ is the relative displacement along axis $i$ of the cross section of the piezoactuator or the piezoplate, $\Psi_{m}=\left\{E_{m}, D_{m}, H_{m}\right.$ is the control parameter, $E_{m}$ is the electric field strength for the voltage control along axis $m, D_{m}$ is the electric induction for the current control along axis $m, H_{m}$ for magnetic field strength control along axis $m, T_{j}$ is the mechanical stress along axis $j, v_{m i}$ is the electromagnetoelastic module, for example, the piezoelectric module, $s_{i j}^{\Psi}$ is the elastic compliance for the control parameter $\Psi=\mathrm{const}$, and the indexes $i=1,2, \ldots, 6 ; j=1,2, \ldots$, $6 ; m=1,2,3$. The main size of the electromagnetoelastic actuator is determined us the working length $l=\{\delta, h, b$ in form the thickness, the height and the width for the longitudinal, transverse and shift piezoeffect.
For the construction the structural diagrams of the electromagnetoelastic actuator is used the wave equation ${ }^{8,10,14}$ for the wave propagation in a long line with damping but without distortions. With using Laplace transform is obtained the linear ordinary second-order differential equation with $p$ parameter. The original problem for the partial differential equation of hyperbolic type using the Laplace transform is reduced to the simpler problem ${ }^{8,14}$ for the linear ordinary differential equation

$$
\frac{d^{2} \Xi(x, p)}{d x^{2}}-\gamma^{2} \Xi(x, p)=0
$$

where $\Xi(x, p)$ is the Laplace transform of the displacement of section of the actuator, $\gamma=p / c^{\Psi}+\alpha$ is the propagation coefficient, $c^{\Psi}$ is the sound speed for the control parameter $\Psi=$ const,$\alpha$ is the damping coefficient, $C$ and $B$ are constants.

The mathematical model and the generalized structural diagram of the electromagnetoelastic actuator for the nano- and microdisplacement ${ }^{7,14}$ on Figure 1 are determined, using method of the mathematical physics for the solution of the wave equation, the boundary conditions and the equation of the electromagnetoelasticity.

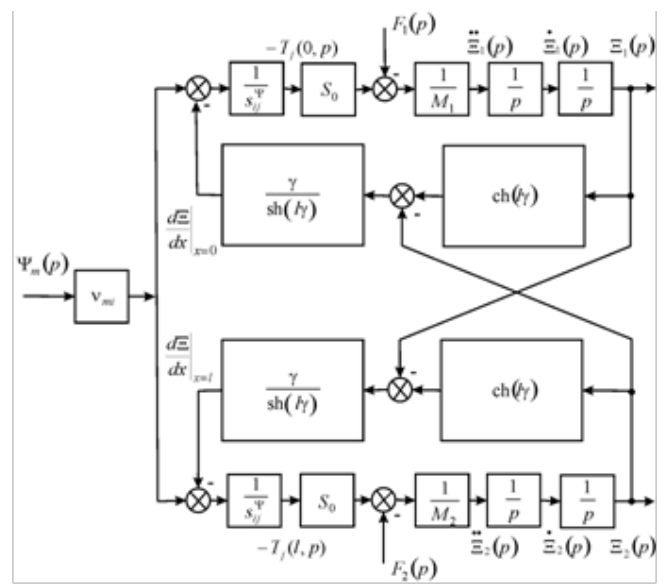

Figure I Generalized structural diagram of electromagnetoelastic actuator for the nano- and microdisplacement.

The generalized structural-parametric model of the electromagnetoelastic actuator has form of the system of the equations 


$$
\begin{aligned}
& \Xi_{1}(p)=\left[1 /\left(M_{1} p^{2}\right)\right] \times \\
& \times\left\{-F_{1}(p)+\left(1 / \chi_{i j}^{\Psi}\right)\left[v_{m i} \Psi_{m}(p)-[\gamma / \operatorname{sh}(l \gamma)]\left[\operatorname{ch}(l \gamma) \Xi_{1}(p)-\Xi_{2}(p)\right]\right]\right\}
\end{aligned}
$$

$$
\begin{aligned}
& \Xi_{2}(p)=\left[1 /\left(M_{2} p^{2}\right)\right] \times \\
& \times\left\{-F_{2}(p)+\left(1 / \chi_{i j}^{\Psi}\right)\left[v_{m i} \Psi_{m}(p)-[\gamma / \operatorname{sh}(l \gamma)]\left[\operatorname{ch}(l \gamma) \Xi_{2}(p)-\Xi_{1}(p)\right]\right]\right\} \\
& \text { where } v_{m i}=\left\{\begin{array}{l}
d_{33}, d_{31}, d_{15} \\
g_{33}, g_{31}, g_{15} \\
d_{33}, d_{31}, d_{15}
\end{array}, \Psi_{m}=\left\{\begin{array}{l}
E_{3}, E_{1} \\
D_{3}, D_{1}, s_{i j}^{\Psi} \\
H_{3}, H_{1}
\end{array}=\left\{\begin{array}{l}
s_{33}^{E}, s_{11}^{E}, s_{55}^{E} \\
s_{33}, s_{11}^{D}, s_{55}^{D} \\
s_{33}, s_{11}^{H}, s_{55}^{H}
\end{array}\right.\right.\right. \\
& c^{\Psi}=\left\{\begin{array}{l}
c^{E} \\
c^{D}, \\
c^{H}
\end{array}, \gamma=\left\{\begin{array}{l}
\gamma^{E} \\
\gamma^{D}, l=\left\{\begin{array}{l}
\delta \\
\gamma^{H}, \chi_{i j}^{\Psi}=s_{i j}^{\Psi} / S_{0} \\
b
\end{array}\right.
\end{array}\right.\right.
\end{aligned}
$$

$v_{m i}$ is the electromagnetoelastic module, $\Psi_{m}=\left\{E_{m}, D_{m}, H_{m}\right.$ is the control parameter, $E_{m}$ is the electric field strength for the voltage control along axis $m, \stackrel{m}{D}$ is the electric induction for the current control along axis $m, H_{m}$ for magnetic field strength control along axis $m, s_{i j}^{\Psi}$ is the elastic compliance, $d_{m i}$ is the piezomodule at the voltage-controlled piezoactuator or the magnetostrictive coefficient for the magnetostrictive actuator, $g_{m i}$ is the piezomodule at the current-controlled piezoactuator, $S_{Q}$ is the cross section area, $M_{y}$, $M_{2}$ are the mass of the load, $\Xi_{1}(p), \Xi_{2}(p)$ and $F_{1}(p), F_{2}(p)$ are the Laplace transforms of the appropriate displacements and the forces on the faces 1,2 . The structural diagrams of the magnetostrictive actuator, the voltage-controlled or current-controlled piezoactuator are determined from the mathematical model of the actuator.

\section{Matrix transfer function}

The matrix transfer function of the electromagnetoelastic actuator $^{8,14,18}$ for the large telescopes, the cosmic telescopes and the adaptive optics is deduced from its mathematical model (3) in the following form

$$
\begin{gathered}
(\Xi(p))=(W(p))(P(p)) \\
(\Xi(p))=\left(\begin{array}{c}
\Xi_{1}(p) \\
\Xi_{2}(p)
\end{array}\right),(W(p))=\left(\begin{array}{lll}
W_{11}(p) & W_{12}(p) & W_{13}(p) \\
W_{21}(p) & W_{22}(p) & W_{23}(p)
\end{array}\right) \\
(P(p))=\left(\begin{array}{c}
\Psi_{m}(p) \\
F_{1}(p) \\
F_{2}(p)
\end{array}\right)
\end{gathered}
$$

where $(\Xi(p))$ is the column-matrix of the Laplace transforms of the displacements for the faces of the electromagnetoelastic actuator, $(W(p))$ is the matrix transfer function, $(P(p))$ the column-matrix of the Laplace transforms of the control parameter and the forces.

\section{Conclusion}

The mathematical model, the structural diagram and transfer functions of the electromagnetoelastic actuator for the large telescopes, the cosmic telescopes and the adaptive optics are obtained. The generalized structural diagram, the transfer functions of the electromagnetoelastic actuator for the nano- and microdisplacement are described the characteristics of the electromagnetoelastic actuator with regard to its physical parameters, external load.

\section{Acknowledgements}

None.

\section{Conflict of interest}

The author declares that there is no conflict of interest.

\section{References}

1. Schultz J, Ueda J, Asada H. Cellular actuators. Oxford: ButterworthHeinemann Publisher; 2017. 382 p.

2. Afonin SM. Absolute stability conditions for a system controlling the deformation of an elecromagnetoelastic transduser. Doklady Mathematics. 2006;74(3):943-948.

3. Zhou S, Yao Z. Design and optimization of a modal-independent linear ultrasonic motor. IEEE Trans Ultrason Ferroelectr Freq Control. 2014;61(3):535-546.

4. Przybylski J. Static and dynamic analysis of a flextensional transducer with an axial piezoelectric actuation. Engineering Structures. 2015;84:140-151.

5. Ueda J, Secord T, Asada HH. Large effective-strain piezoelectric actuators using nested cellular architecture with exponential strain amplification mechanisms. IEEE/ASME Transactions on Mechatronics. 2010;15(5):770-782.

6. Karpelson M, Wei GY, Wood RJ. Driving high voltage piezoelectric actuators in microrobotic applications. Sensors and Actuators A: Physical. 2012;176:78-89.

7. Afonin SM. Block diagrams of a multilayer piezoelectric motor for nanoand microdisplacements based on the transverse piezoeffect. Journal of Computer and Systems Sciences International. 2015;54(3):424-439.

8. Afonin SM. Structural parametric model of a piezoelectric nanodisplacement transduser. Doklady Physics. 2008;53(3):137-143.

9. Afonin SM. Solution of the wave equation for the control of an elecromagnetoelastic transduser. Doklady Mathematics. 2006;73(2):307-313.

10. Cady WG. Piezoelectricity: An introduction to the theory and applications of electromechancial phenomena in crystals. New York, London: McGraw-Hill Book Company; 1946. 806 p.

11. Physical acoustics: Principles and methods. Vol.1. Part A. Methods and devices. Mason W. editor, New York: Academic Press; 1964. 515 p.

12. Zwillinger D. Handbook of differential equations. Boston: Academic Press; 1989. 673 p.

13. Afonin SM. Structural-parametric model and transfer functions of electroelastic actuator for nano- and microdisplacement. Chapter 9 in Piezoelectrics and nanomaterials: Fundamentals, developments 
and applications. Parinov IA. editor, New York: Nova Science. 2015. 225-242 p.

14. Afonin SM. A structural-parametric model of electroelastic actuator for nano- and microdisplacement of mechatronic system. Chapter 8 in Advances in nanotechnology. Volume 19. Bartul Z, Trenor J editors, New York: Nova Science; 2017. 259-284 p.

15. Afonin SM. Nano- and micro-scale piezomotors. Russian Engineering Research. 2012;32(7-8):519-522.

16. Afonin SM. Elastic compliances and mechanical and adjusting characteristics of composite piezoelectric transducers. Mechanics of Solids. 2007;42(1):43-49.

17. Afonin SM. Stability of strain control systems of nano-and microdisplacement piezotransducers. Mechanics of Solids. 2014;49(2):196-207.

18. Afonin SM. Structural-parametric model electromagnetoelastic actuator nanodisplacement for mechatronics. International Journal of Physics. 2017;5(1):9-15.

19. Afonin SM. Structural-parametric model of piezoactuator nano- and microdisplacement for nanoscience. AASCIT Journal of Nanoscience. 2017;3(3):12-18.

20. Afonin SM. Solution wave equation and parametric structural schematic diagrams of electromagnetoelastic actuators nano- and microdisplacement. International Journal of Mathematical Analysis and Applications. 2016;3(4):31-38.

21. Afonin SM. Structural-parametric model of electromagnetoelastic actuator for nanomechanics. Actuators. 2018;7(1):1-9.

22. Afonin SM. Structural-parametric models and transfer functions of electromagnetoelastic actuators nano- and microdisplacement for mechatronic systems. International Journal of Theoretical and Applied Mathematics. 2016;2(2):52-59. 